\title{
Diagnosis of polyglutamine spinocerebellar ataxias by polymerase chain reaction amplification and Sanger sequencing
}

\author{
CHANGQIANG CHEN, XUQIAN FANG and SHUNCHANG SUN \\ Department of Laboratory Medicine, Ruijin Hospital North, \\ Shanghai Jiao Tong University School of Medicine, Shanghai 201801, P.R. China
}

Received January 6, 2018; Accepted May 9, 2018

DOI: $10.3892 / \mathrm{mmr} .2018 .9043$

\begin{abstract}
Spinocerebellar ataxia (SCA) is a group of genetic diseases of the nervous system with genetic and clinical heterogeneity. SCA is often caused by an expanded CAG repeat sequence in the encoding protein. Genetic testing is necessary to diagnose and classify the types of SCA. Next-generation DNA sequencing usually generates a high error rate for insertion or deletion mutations, so it is unhelpful for classifying the types of SCA. In the present study, a Chinese SCA pedigree was preliminarily diagnosed with SCA1 using polymerase chain reaction (PCR) amplification. The propositus and his three younger siblings were diagnosed with SCA1 as a result of the identification of the length of the expanded CAG repeat sequence in the ATXN1 gene performed using Sanger sequencing. The current study presents a convenient and efficient method to identify causative mutations for polyglutamine SCA using PCR amplification followed by Sanger sequencing.
\end{abstract}

\section{Introduction}

Spinocerebellar ataxias (SCAs), a genetically heterogeneous group of disorders, are inherited in an autosomal-dominant pattern. Currently, over 30 different types of SCA have been reported, however only $\sim 50 \%$ of causative mutations have been identified (1). The majority of SCA cases are caused by an abnormally expanded CAG repeat sequence that leads to the expansion of a tract of polyglutamine residues in the encoded proteins (2). The SCAs caused by the CAG repeat expansion mutation are classified as polyglutamine SCAs. These include SCA1, SCA2, SCA3, SCA6, SCA7, SCA12, SCA17, and dentatorubral-pallidoluysian atrophy (DRPLA), in which the expansion within the glutamine-encoding CAG trinucleotide repeats accounts for SCA (3). Healthy individuals usually

Correspondence to: Dr Shunchang Sun, Department of Laboratory Medicine, Ruijin Hospital North, Shanghai Jiao Tong University School of Medicine, 999 Xiwang Road, Shanghai 201801, P.R. China E-mail: shunchangsun@aliyun.com

Key words: spinocerebellar ataxia, diagnosis; polymerase chain reaction amplification, Sanger sequencing carry the heterozygous $\mathrm{CAG}$ repeats at a number below the predicted pathological threshold. SCA8, probably arising from a non-coding CTG repeat expansion is also grouped with polyglutamine SCAs (4). The threshold of CAG/CTG expansions that determine disease carrier status varies between the different types of SCA (5). Several methods have been used for detecting polyglutamine SCA mutations including fluorescence polymerase chain reaction (PCR), denaturing polyacrylamide gel electrophoresis of DNA, next-generation DNA sequencing and direct sequencing (6). However, these methods have some limitations when applied to a clinical laboratory setting (7). Therefore, there is a need to develop a simple and rapid method for the detection of CAG repeat mutations. PCR amplification of the CAG repeat mutations followed by Sanger sequencing is a simple and inexpensive method for detection. In the current study, the authors present a convenient method for screening causative mutations for polyglutamine SCA using PCR amplification followed by Sanger sequencing. A Chinese SCA1 pedigree was identified using this method.

\section{Materials and methods}

Pedigree. The study was approved by the ethics committee of Shenzhen Baoan Hospital, Southern Medical University (Shenzhen, China). Written informed patient consent was obtained before initiating the study. The pedigree investigated in the present study is shown in Fig. 1. The propositus (IV-3) a 24-year-old male individual phenotypically characterized by a slowly progressive incoordination of gait, associated with poor coordination of hands, was referred to the Department of Neurology due to primary difficulty walking. The propositus complained of slow walking and unsteady gait from the age of 18. The clinical features of the propositus included limb ataxia, dysarthria, dysphagia, action tremors, increased tendon reflexes and handwriting difficulties on examination. Brain magnetic resonance imaging (data not shown) indicated prominent atrophy of the cerebellum and brainstem. The family was diagnosed with SCA by a clinical neurologist according to neuroimaging, family history and physical examination. The age of onset was defined according to the early signs of gait disturbance and slurred speech described by the family members in the SCA pedigree. Genomic DNA was extracted from the peripheral leukocytes 
of the 8 subjects given serial numbers in the SCA pedigree and 205 normal controls using standard protocol (8). Three subjects (IV-5, 7 and 8 ) were asymptomatic causative SCA1 gene carriers at gene diagnosis in the pedigree.

Screening PCR. Polyglutamine SCA includes SCA1, SCA2, SCA3, SCA6, SCA7, SCA8, SCA12, SCA17 and DRPLA. A total of 9 fragments including CAG/CTG expansion sequences were amplified by PCR using primer sets shown in Table I. First, 100 ng of genomic DNA was amplified by PCR in a $50 \mu 1$ reaction volume containing $10 \mathrm{mM}$ Tris- $\mathrm{HCl},(\mathrm{pH}$ 8.3), $2.5 \mathrm{mM} \mathrm{MgCl}_{2}, 50 \mathrm{mM} \mathrm{KCl}, 200 \mathrm{M}$ of each dNTP, $2 \mathrm{U}$ Taq polymerase, and $0.5 \mathrm{M}$ each primer (Table I). Following denaturation at $95^{\circ} \mathrm{C}$ for $7 \mathrm{~min}$, amplification was performed at $95^{\circ} \mathrm{C}$ for 50 seconds, annealing for $50 \mathrm{sec}$ under different temperatures as described in Table I, and $72^{\circ} \mathrm{C}$ for $60 \mathrm{sec}$ for 35 cycles followed by a final extension at $72^{\circ} \mathrm{C}$ for $7 \mathrm{~min}$. After amplification, electrophoresis was performed in a $2 \%$ agarose gel $(100 \mathrm{~V}, 45 \mathrm{~min})$ to detect the amplified DNA fragments, then agarose gel was exposed under a UV light imager.

Sanger sequencing. Following purification, amplified PCR fragments were subjected to bidirectional direct and cycle sequencing. Direct sequencing was performed using the ABI3730 Genetic Analyzer (Applied Biosystems; Thermo Fisher Scientific, Inc., Waltham, MA, USA). Cycle sequencing was performed using Stratagene's RoboCycler Gradient (Stratagene; Agilent Technologies, Inc., Santa Clara, CA, USA) 96 temperature cycler with Hot Top Assembly. The DNA polymerase used in sequencing was Platinum SuperFi DNA Polymerase (Invitrogen, Thermo Fisher Scientific, Inc.) which combines high fidelity and high sequence accuracy. All sequencing of PCR fragments was performed more than twice, the identical sequencing results were regarded as the number of CAG triplet repeats carried by individuals.

\section{Results}

Mutation screening. The mutation screening results of SCA1, SCA2, SCA3, SCA6, SCA7, SCA8, SCA12, SCA17 and DRPLA are presented in Fig. 2 for the propositus in the SCA pedigree and a healthy subject. Two amplification fragments of distinctly different sizes were observed for the propositus using the primer sets to amplify the CAG repeats in the ATXNI gene and two fragments of nearly the same size or only a fragment of amplification were found using other primer sets. Two fragments of nearly the same size or only a fragment of amplification were seen for the healthy subject using each pair of PCR primers. Mutation screening suggested that the propositus was a SCA1 patient. The screening PCR detected two fragments of distinctly different sizes in 4 out of the 8 tested individuals including the propositus using the given set of primers to amplify the SCA1 CAG expansion (Fig. 3). The three siblings of the propositus (IV-5, IV-7 and V-8) were asymptomatic causative SCA1 gene carriers as determined by gene mutation analysis. Sanger sequencing was needed in order to count the number of CAG repeats in the ATXN1 gene in the SCA pedigree for accurate diagnosis. The other four members (II-1, III-4, IV-1 and IV-6) were healthy subjects because only two fragments of nearly the same size were found.

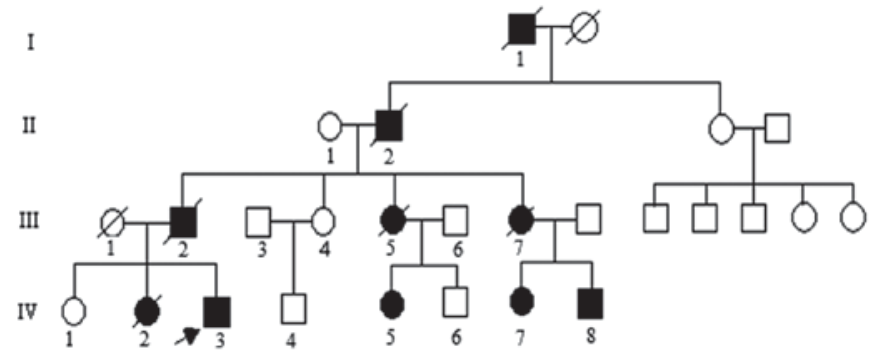

Figure 1. Pedigree of the family with SCA. Squares indicate males; circles indicate females; an arrow indicates the propositus. Slash marks indicate subjects who are deceased. Roman numerals indicate generations, and Arabic numbers indicate subjects. SCA, spinocerebellar ataxia.

Sanger sequencing. With Sanger sequencing, 67 CAG trinucleotide repeat genotypes were detected in 205 normal healthy subjects (Table II). The normal range of the SCA1 CAG trinucleotide repeats was 7-38 repeats, with the common repeat being 23-33 repeats in the Chinese population (Fig. 4). The normal range of CAG repeats was similar to that reported for other ethnic populations. Usually normal alleles containing 18-38 CAG repeats were interrupted by 1-3 CAT interruptions, however a few alleles containing 7-23 repeats were not interrupted. In the present study, a CAG triplet repeat (length 61) was detected for the propositus (IV-3). A repeat length of 51 (IV-7) and 50 (in both IV-5 and IV-8) was observed in the three younger siblings for the SCA1 pedigree. The other two subjects (IV-1 and IV-6) carried a normal range of CAG repeats (28-33) in the ATXN1 gene (Table III). The larger the CAG repeat, the earlier the age of onset for SCA1 (Table IV). The three siblings with 50 or 51 triplet repeats had no SCA1 symptoms, which indicated a possible late onset. The family members IV-5, IV-7 and IV-8 could be diagnosed with SCA1 according to the sequencing results, while the subjects IV-1 and IV-6 could be considered healthy individuals.

\section{Discussion}

Polyglutamine SCAs represent a genetically heterogeneous group of disorders involving the nervous system with a dominant pattern of inheritance. These SCAs are caused by the expansion of a CAG/CTG triplet repeat (9). Diagnosis of polyglutamine SCA requires clinical assessment for the presence of ataxia and onset age, a family history, and the clinical characteristics. The diagnosis is only the beginning; a confirmative genetic study should be performed to classify the type of SCA for genetic counseling and family planning (10). This would gradually reduce the incidence of the SCA. Healthy subjects often carry the heterozygous CAG repeats below the pathological threshold, therefore, two PCR fragments of nearly the same size are usually found using agarose gel electrophoresis. Two fragments of distinctly different sizes indicate the pathologically expanded CAG repeat sequence is present. The present study demonstrated a convenient molecular analysis technique for accurate diagnosis of polyglutamine SCA by PCR amplification and Sanger sequencing. The diagnosis of SCA1 was suspected for the family of the propositus with the CAG expansion in the ATXN1 gene by PCR amplification. The CAG triplet 
Table I. Sequences of oligonucleotide primer sets for polymerase chain reaction.

\begin{tabular}{|c|c|c|c|}
\hline Primer & Sequence $\left(5^{\prime}-3^{\prime}\right)$ & Annealing temperature $\left({ }^{\circ} \mathrm{C}\right)$ & Fragment size of the wild-type allele \\
\hline SCA1-F & ggtcctcccaatacagtgga & 60 & $364-403$ \\
\hline SCA1-R & ttctgcggagaactggaaat & & \\
\hline SCA2-F & ctccgectcagactgttttg & 61 & $810-837$ \\
\hline SCA2-R & ctgaccatcgecgetacc & & \\
\hline SCA3-F & tctgtatcagactaactgctcttgc & 59 & $369-438$ \\
\hline SCA3-R & gagggaatgaagaataatgtaaagc & & \\
\hline SCA6-F & tcccgtgtctcctttgattt & 59 & $378-411$ \\
\hline SCA6-R & gaccegectctccatcct & & \\
\hline SCA7-F & taggagcggaaagaatgtcg & 56 & $280-310$ \\
\hline SCA7-R & cccagcatcacttcaggact & & \\
\hline SCA8-F & gcagtatgaggaagtatggaaaaa & 59 & $230-335$ \\
\hline SCA8-R & ttctgactcccagcttccac & & \\
\hline SCA12-F & ccactgcagcaaagagca & 59 & $280-355$ \\
\hline SCA12-R & ggaatgagggtgctggtc & & \\
\hline SCA17-F & gacccacagcctattcaga & 60 & $196-247$ \\
\hline SCA17-R & gcctgaggttccctgtgtt & & \\
\hline DRPLA-F & ccaccacctcctccetatg & 60 & $209-257$ \\
\hline DRPLA-R & agtgggtggggaaatgct & & \\
\hline
\end{tabular}

SCA, spinocerebellar ataxia; DRPLA, dentatorubral-pallidoluysian atrophy; F, forward; R, reverse.

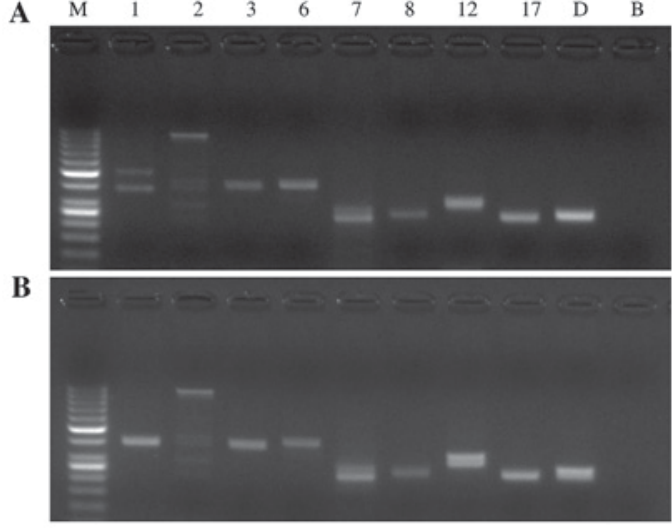

Figure 2. Screening PCR for SCA1, SCA2, SCA3, SCA6, SCA7, SCA8, SCA12, SCA17 and DRPLA for the (A) propositus in the SCA pedigree and (B) the healthy subject. Lane M: Molecular weight marker DNA ladder $(1,000,900,800,700,600,500,400,300,250,200,150,100$ and $50 \mathrm{bp})$; lane 1-D: The amplified fragments with primer sets for SCA1, SCA2, SCA3, SCA6, SCA7, SCA8, SCA12, SCA17, and DRPLA, respectively; lane B: blank. PCR, polymerase chain reaction; SCA, spinocerebellar ataxia; DRPLA, dentatorubral-pallidoluysian atrophy

repeat expansion was found in the propositus and his three younger siblings in the ATXN1 gene. PCR is a relatively simple and inexpensive tool that can be used in most clinical laboratories, and agarose gel electrophoresis of the PCR products is easy to perform. Screening for polyglutamine SCAs by PCR is a rapid and efficient method compared with other mutation detection methods. The definitive diagnosis of SCA1 depended on the results of Sanger sequencing of the CAG repeat expansion in the ATXN1 gene. A total of $61 \mathrm{CAG}$ triplet repeats were detected for the propositus who

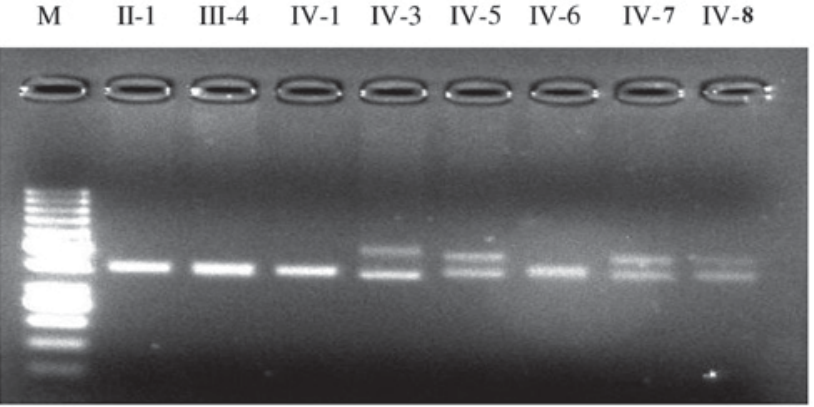

Figure 3. Screening PCR for SCA1 for the SCA pedigree. Lane M: molecular weight marker DNA ladder. Lanes marked with roman numerals indicated the serial number of subjects. The serial number was given to the individuals according to The American Society of Human Genetics. The Roman numerals indicated generation, the Arabic numerals indicated the number in the same generation. PCR, polymerase chain reaction; SCA, spinocerebellar ataxia; DRPLA, dentatorubral-pallidoluysian atrophy.

presented with initial ataxia symptoms and signs at the age of 18. The three younger siblings of the propositus carrying 51 or 50 CAG repeats in the ATXN1 gene showed no symptoms or signs of ataxia at the age of 22-28 years for the SCA1 pedigree. Three subjects carrying the $\mathrm{CAG}$ repeat expansion were diagnosed with SCA1 according to the sequencing results.

The onset age is inversely correlated with the number of CAG triplet repeats, the triplet repeat number is also associated with the severity of the disease. The larger the CAG triplet repeat number, the more serious the disease (11). In the present study, a pedigree showing genetic anticipation in SCA was investigated. Genetic anticipation in the age at which onset was observed in the past three generations for 


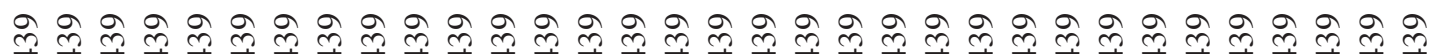
志志志志志志志志志志志志志志志志志志志志志志志志志志

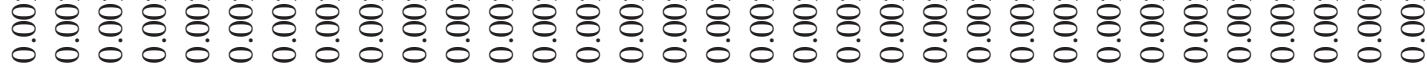

离

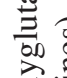

m

㻤.

言

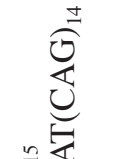

(2)

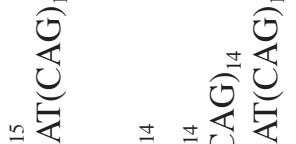

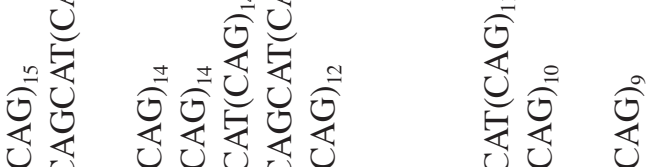

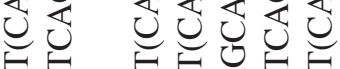

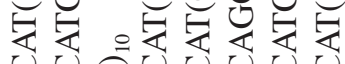

论

Oิ

论

$\stackrel{8}{0}$

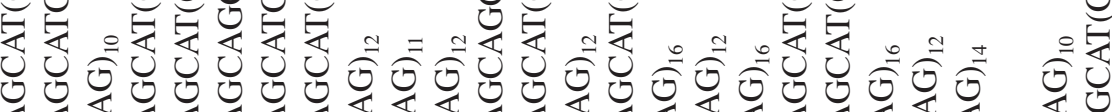

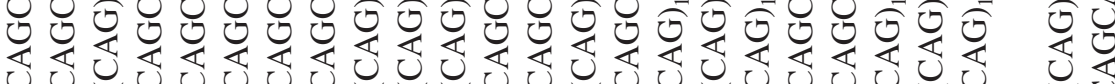

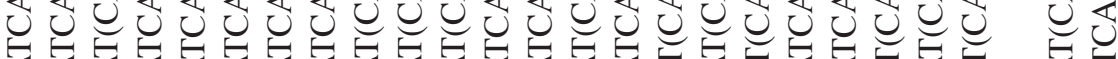

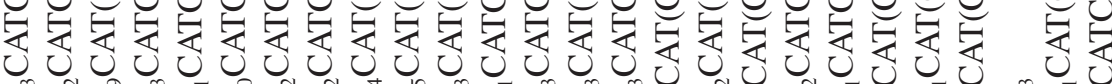

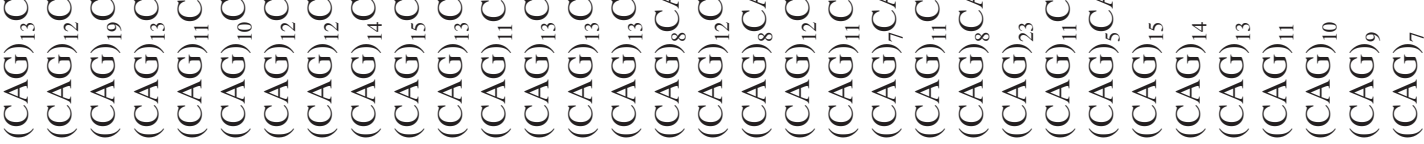

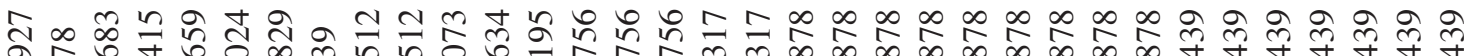

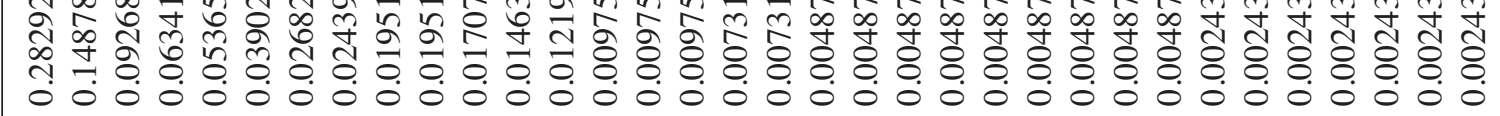

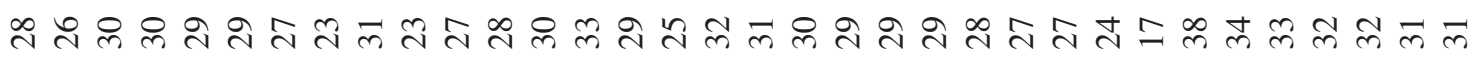

O0.0

过过茛过

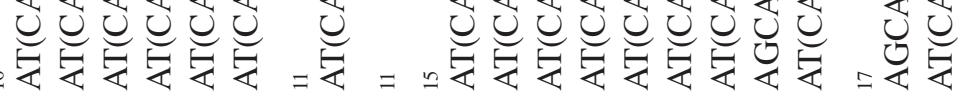

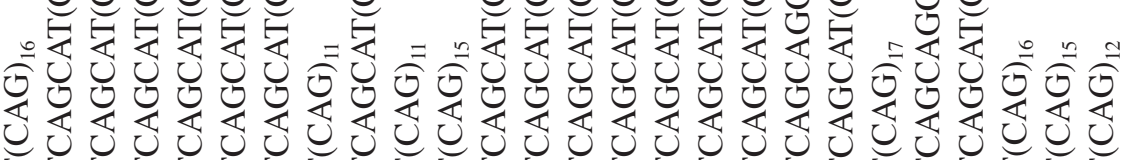

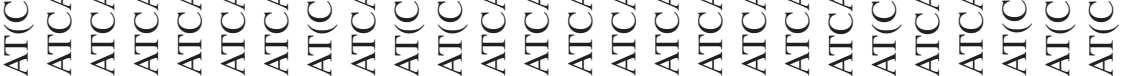

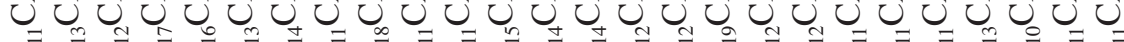

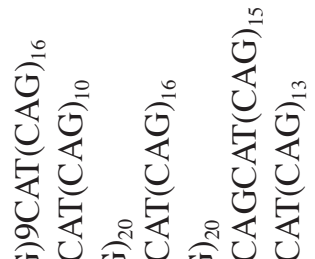


Table III. CAG repeats in the ATXN1 gene in the spinocerebellar ataxia pedigree.

\begin{tabular}{llc}
\hline Subjects & \multicolumn{1}{c}{ Allele } & Number of polyglutamines (histidines) \\
\hline II-1 & $(\mathrm{CAG})_{16}$ CATCAGCAT $(\mathrm{CAG})_{10} /(\mathrm{CAG})_{17}$ CATCAGCAT(CAG) $)_{10}$ & $29 / 30$ \\
III-4 & $(\mathrm{CAG})_{16}$ CATCAGCAT $(\mathrm{CAG})_{10} /(\mathrm{CAG})_{13}$ CATCAGCAT(CAG $)_{10}$ & $29 / 26$ \\
IV-1 & $(\mathrm{CAG})_{16}$ CATCAGCAT $(\mathrm{CAG})_{10} /(\mathrm{CAG})_{11}$ CAT $(\mathrm{CAG})_{16}$ & $29 / 28$ \\
IV-3 & $(\mathrm{CAG})_{13}$ CATCAGCAT $(\mathrm{CAG})_{10} /(\mathrm{CAG})_{61}$ & $26 / 61$ \\
IV-5 & $(\mathrm{CAG})_{12}$ CATCAGCAT $(\mathrm{CAG})_{15} /(\mathrm{CAG})_{50}$ & $30 / 50$ \\
IV-6 & $(\mathrm{CAG})_{15}$ CATCAGCAT $(\mathrm{CAG})_{15} /(\mathrm{CAG})_{17}$ CATCAGCAT $(\mathrm{CAG})_{10}$ & $33 / 30$ \\
IV-7 & $(\mathrm{CAG})_{12}$ CATCAGCAT $(\mathrm{CAG})_{15} /(\mathrm{CAG})_{51}$ & $30 / 51$ \\
IV-8 & $(\mathrm{CAG})_{12}$ CATCAGCAT $(\mathrm{CAG})_{15} /(\mathrm{CAG})_{50}$ & $30 / 50$ \\
\hline
\end{tabular}

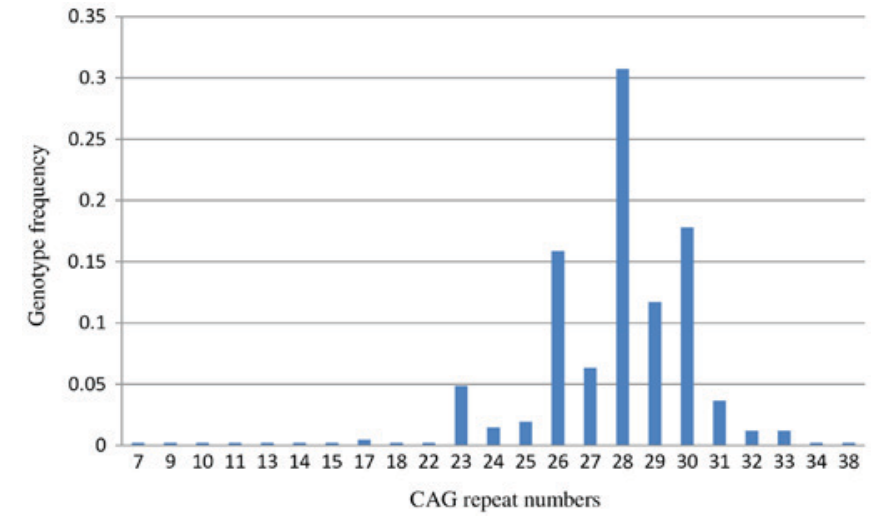

Figure 4. Genotype frequency of different CAG trinucleotide repeats in the $A T X N 1$ gene in healthy Chinese subjects.

the family according to the families' recollection (Table IV). The mechanism of genetic anticipation in SCA is the intergenerational unstable amplification of CAG triplet repeat sequences. The greater the CAG repeat number increases with the generation, the more the age-at-onset decreases in successive generations (12). Genetic anticipation tends to be more prominent when the CAG expansion comes from paternal inheritance compared with maternal inheritance (13). The different onset age between the propositus and his three younger siblings should be caused by a distinctive inheritance pattern. The propositus obtained the causative mutation of CAG repeat expansion from his father who died of SCA1 at the age of 35. Three siblings of the propositus carrying 50-51 CAG repeats showed no symptoms or signs of SCA1 at the age of 22-28 years as a result of maternal inheritance. The phenotype of the disease may be present $\sim 10$ years later for the three younger siblings carrying 50-51 CAG repeats, according to the correlation between the length of CAG repeat expansion and age at disease onset, although statistical analyses were not performed. Certainly, the age-at-onset and phenotype of the disease can be affected by regulating genetic and environmental factors (14). Thus, Sanger sequencing of the fragments of the trinucleotide repeat sequence is helpful to predict the age-at-onset and phenotype of SCA. In the Chinese population, gene mutations in ATXN3 are the most common, followed by SCA2, SCA1, SCA7, SCA6, and SCA12 (15). In the present study, there was evidence of genetic anticipation, which could be caused
Table IV. Age of the onset in the spinocerebellar ataxia 1 pedigree.

\begin{tabular}{lcc}
\hline Subjects & $\begin{array}{c}\text { Age of the onset } \\
\text { (Years) }\end{array}$ & $\begin{array}{c}\text { Number of polyglutamines } \\
\text { (histidines) }\end{array}$ \\
\hline I-1 & 37 & N/A \\
II-2 & 34 & N/A \\
III-2 & 28 & N/A \\
III-5 & 26 & N/A \\
III-7 & 29 & N/A \\
IV-2 & 22 & N/A \\
IV-3 & 18 & $26 / 61$ \\
IV-5 & Asymptomatic & $30 / 50$ \\
IV-7 & Asymptomatic & $30 / 51$ \\
IV-8 & Asymptomatic & $30 / 50$ \\
\hline
\end{tabular}

N/A, not applicable (DNA was not obtained).

by an abnormally expanded CAG repeat sequence in the pedigree. The family was affected by a polyglutamine SCA. The SCA1 was a preliminary diagnosis for the family by PCR amplification. Accurate diagnosis of 4 cases with SCA1 was performed using Sanger sequencing of the CAG repeat expansion in the ATXN1 gene, which was helpful to infer the age-at-onset. The results of the present study suggest that PCR amplification followed by Sanger sequencing is a useful tool to diagnose polyglutamine SCA.

\section{Acknowledgments}

Not applicable.

\section{Funding}

The present study was supported by the National Natural Science Foundation of China (31571294).

\section{Availability of data and materials}

All data generated or analyzed during this study are included in this published article. 


\section{Authors' contributions}

$\mathrm{CC}$ analyzed the patient data and performed screening PCR. XF performed Sanger sequencing. SS designed the study and was a major contributor in writing the manuscript. All authors read and approved the final manuscript.

\section{Ethics approval and consent to participate}

The study was approved by the ethics committee of Shenzhen Baoan Hospital, Southern Medical University. Written informed patient consent was obtained before initiating the study.

\section{Consent for publication}

The patient has provided written informed consent for the publication of any associated data.

\section{Competing interests}

The authors declare that they have no competing interests.

\section{References}

1. Nibbeling EAR, Duarri A, Verschuuren-Bemelmans CC, Fokkens MR, Karjalainen JM, Smeets CJLM, de Boer-Bergsma JJ, van der Vries G, Dooijes D, Bampi GB, et al: Exome sequencing and network analysis identifies shared mechanisms underlying spinocerebellar ataxia. Brain 140: 2860-2878, 2017.

2. Dell'Orco JM, Pulst SM and Shakkottai VG: Potassium channel dysfunction underlies Purkinje neuron spiking abnormalities in spinocerebellar ataxia type 2. Hum Mol Genet 26: 3935-3945, 2017

3. Sutton JR, Blount JR, Libohova K, Tsou WL, Joshi GS, Paulson HL, Costa MDC, Scaglione KM and Todi SV: Interaction of the polyglutamine protein ataxin-3 with $\operatorname{Rad} 23$ regulates toxicity in drosophila models of spinocerebellar ataxia type 3 . Hum Mol Genet 26: 1419-1431, 2017.
4. Terada T, Kono S, Konishi T, Miyajima H and Ouchi Y: Altered GABAergic system in the living brain of a patient with spinocerebellar ataxia type 8. J Neurol 260: 3164-3166, 2013.

5. Tezenas du Montcel S, Durr A, Bauer P, Figueroa KP, Ichikawa Y, Brussino A, Forlani S, Rakowicz M, Schöls L, Mariotti C, et al: Modulation of the age at onset in spinocerebellar ataxia by CAG tracts in various genes. Brain 137: 2444-2455, 2014.

6. Li M, Pang SY, Song Y, Kung MH, Ho SL and Sham PC: Whole exome sequencing identifies a novel mutation in the transglutaminase 6 gene for spinocerebellar ataxia in a Chinese family. Clin Genet 83: 269-273, 2013.

7. Gould Rothberg BE and Rothberg JM: Massively parallel ('next-generation') DNA sequencing. Clin Chem 61: 997-998, 2015.

8. Miller SA, Dykes DD and Polesky HF: A simple salting out procedure for extracting DNA from human nucleated cells. Nucleic Acids Res 16: 1215, 1988.

9. Shakkottai VG and Fogel BL: Clinical neurogenetics: Autosomal dominant spinocerebellar ataxia. Neurol Clin 31: 987-1007, 2013.

10. Melo AR, Ramos A, Kazachkova N, Raposo M, Bettencourt BF Rendeiro AR, Kay T, Vasconcelos J, Bruges-Armas J and Lima M: Triplet repeat primed PCR (TP-PCR) in molecular diagnostic testing for spinocerebellar ataxia type 3 (SCA3). Mol Diagn Ther 20: 617-622, 2016.

11. Scoles DR, Meera P, Schneider MD, Paul S, Dansithong W, Figueroa KP, Hung G, Rigo F, Bennett CF, Otis TS and Pulst SM: Antisense oligonucleotide therapy for spinocerebellar ataxia type 2. Nature 544: 362-366, 2017.

12. Lai S, O'Callaghan B, Zoghbi HY and Orr HT: 14-3-3 Binding to ataxin-1(ATXN1) regulates its dephosphorylation at Ser-776 and transport to the nucleus. J Biol Chem 286: 34606-34616, 2011.

13. McMurray CT: Mechanisms of trinucleotide repeat instability during human development. Nat Rev Genet 11: 786-799, 2010.

14. Chatterjee N, Lin Y, Santillan BA, Yotnda P and Wilson JH: Environmental stress induces trinucleotide repeat mutagenesis in human cells. Proc Natl Acad Sci USA 112: 3764-3769, 2015.

15. Wang J, Shen L, Lei L, Xu Q, Zhou J, Liu Y, Guan W, Pan Q, Xia K, Tang B and Jiang H: Spinocerebellar ataxias in mainland China: an updated genetic analysis among a large cohort of familial and sporadic cases. Zhong Nan Da Xue Xue Bao Yi Xue Ban 36: 482-489, 2011. 